\title{
Effect of different directions of attentional shift on inhibition of return in three-dimensional space
}

\author{
Aijun Wang ${ }^{1} \cdot$ Xiaole Liu ${ }^{1,2} \cdot$ Qi Chen ${ }^{3,4} \cdot$ Ming Zhang ${ }^{1,2}$
}

Published online: 12 January 2016

(C) The Psychonomic Society, Inc. 2016

\begin{abstract}
When attention is oriented to a peripheral cue, the processing of nearby stimuli is facilitated. This brief period of facilitation is followed by a long-lasting inhibitory effect, during which there is a delayed response to stimuli presented at a previously cued location. Although the mechanisms underlying the facilitatory effect of attentional orienting/reorienting in threedimensional (3-D) space have been documented, there is not yet consensus as to how attention orients/reorients in depth during the later inhibitory phase (i.e., inhibition of return [IOR]). In the present study, by incorporating the Posner exogenous cueing paradigm into a virtual 3-D environment, we aimed to investigate whether an IOR effect occurs when attention orients and reorients at the uncued depth in the same hemispace, and whether the IOR effects are the same or different when attention orients/reorients along different trajectories in 3-D space. Our results showed asymmetrical spatial IOR effects when attention was oriented/ reoriented at the uncued depth in the same hemispace. Spatial IOR was depth-specific when targets appeared in the near depth plane, whereas it was not depth-specific when targets appeared in
\end{abstract}

\section{Qi Chen}

qi.chen27@gmail.com

Ming Zhang

psyzm@suda.edu.cn

1 Research Center for Psychological and Behavioral Science, Soochow University, 215123 Suzhou, People's Republic of China

2 Department of Psychology, Soochow University, Suzhou 215123, People's Republic of China

3 Center for Studies of Psychological Application and School of Psychology, South China Normal University, 510631 Guangzhou, People's Republic of China

4 Epilepsy Center, Guangdong 999 Brain Hospital, Guangzhou 510631, People's Republic of China the far depth plane. Apart from these results, we also found that attention oriented/reoriented at the same depth but in a different hemispace experienced a reduction in IOR size, thus indicating that the direction-specific spatial IOR mechanisms when attention orients/reorients along different trajectories are different. Taken together, our results suggest that spatial IOR is not entirely "depth-blind," and that the ecological importance of the 3-D world influences the direction of attentional shifts of spatial IOR in 3-D space.

Keywords Three-dimensional (3-D) space · Attentional orienting/reorienting $\cdot$ Inhibition of return (IOR)

Studies of visuospatial attention in two-dimensional (2-D) space have shown that when attention is oriented to a peripheral cue, if the stimulus onset asynchrony between the cue and target is less than $300 \mathrm{~ms}$, processing of a target appearing at a precued peripheral location is facilitated (as compared with the response to a target appearing at a novel location). A brief period of facilitation is followed by a long-lasting inhibitory effect in which there is a delayed response to stimuli presented at the previously cued location. This long-lasting inhibitory effect is called "inhibition of return" (IOR; Posner \& Cohen, 1984; see Klein, 2000).

Studies of visuospatial attention have primarily focused on 2-D space (Corbetta, Kincade, Lewis, Snyder, \& Sapir, 2005; Corbetta, Kincade, Ollinger, McAvoy, \& Shulman, 2000; Corbetta, Patel, \& Shulman, 2008; Corbetta \& Shulman, 2002; Lupiáñez et al., 2004; Mayer, Seidenberg, Dorflinger, \& Rao, 2004; Spence, Lloyd, McGlone, Nicholls, \& Driver, 2000; Zhang, Zhou, \& Zhang, 2012); however, though these 2-D spatial representations show us how attentional orienting responds to the directions of objects, they tell us little about how it is affected by objects' distances. Humans live in a 
three-dimensional (3-D) world, and the third dimension, depth, is a neglected topic in cognitive research, despite the fact that it is crucially important for understanding how we guide our actions. Previous studies have shown that an essential component of primate visual function is the ability to extract and represent 3-D shape and depth from information in 2-D retinal images (Hayman, Verriotis, Jovalekic, Fenton, \& Jeffery, 2011; Janssen, Vogels, \& Orban, 2000; Sereno, Trinath, Augath, \& Logothetis, 2002). Therefore, attentional orienting/reorienting in 3-D space is particularly important for guiding our behavior. Although the ability to extract structures and obtain information about the spatial position of objects from 3-D space has been well-documented, the question of how attentional orienting/reorienting among these reconstructed objects is performed remains controversial.

Previous studies have documented evidence of the mechanisms underlying the facilitatory effect of attentional orienting in 3-D space (Andersen \& Kramer, 1993; Chen, Weidner, Vossel, Weiss, \& Fink, 2012; de Gonzaga Gawryszewski, Riggio, Rizzolatti, \& Umiltà, 1987; Downing \& Pinker, 1985; Maringelli, McCarthy, Steed, Slater, \& Umiltà, 2001). Behaviorally, they showed that fewer benefits exist for shifting attention from near to far locations than for shifting from far to near locations. It was thus proposed that attentional resources are allocated in depth in a viewer-centered fashion, with the maximum processing resources being allocated to stimuli near the observer's body (de Gonzaga Gawryszewski et al., 1987; Downing \& Pinker, 1985; Maringelli et al., 2001). Furthermore, to survive everyday life while navigating through the 3-D world around us, one of the most important behaviors from a biological/ecological perspective involves the rapid redirection of our attention to potentially threatening or rewarding stimuli that unexpectedly invade spaces closer to us. The depth of a potentially threatening/rewarding stimulus relative to the observer is crucial: Stimuli that unexpectedly approach observers instantaneously demand a shift of attention toward them in order to secure survival. In contrast, stimuli far from observers may be less attention-demanding (de Gonzaga Gawryszewski et al., 1987; Downing \& Pinker, 1985; Graziano \& Cooke, 2006; Previc, 1998). Similarly, in a recent study Chen et al. (2012) constructed a virtual 3-D world by presenting targets either close to or far from participants, in an adapted version of the Posner spatial-cueing paradigm, to investigate the mechanisms underlying visuospatial orienting/reorienting in depth during the earlier, facilitatory phase. They found that attentional reorienting to objects unexpectedly presented closer to observers in the unattended hemispace was faster than reorienting to unexpected objects farther away.

The biphasic hypothesis that attentional orienting/ reorienting consists of an early facilitation followed by a later inhibition has been criticized in several studies (Berlucchi, Chelazzi, \& Tassinari, 2000; Cheal \& Chastain, 2002; Chica
\& Lupiáñez, 2009; Chica, Lupiáñez, \& Bartolomeo, 2006; Kalogeropopulou, Woodruff, \& Vivas, 2015; Klein, 2000; Maruff, Yucel, Danckert, Stuart, \& Currie, 1999; Wright \& Richard, 1996). These researchers suggested that inhibition and facilitation might be two simultaneous, independent processes that overlap in time (Berlucchi et al., 2000; Kalogeropopulou et al., 2015). In addition, when peripheral cues are spatially predictive of target appearance at their same spatial location, an initial facilitation at the cued location is behaviorally measured, whereas no IOR is behaviorally observed (Chica \& Lupiáñez, 2009; Chica et al., 2006). Therefore, typically, when peripheral cues are spatially nonpredictive of target appearance within the context of the spatial cueing/IOR tasks, it is widely accepted that the description of a facilitation and an inhibition is done in accordance with the biphasic hypothesis (Chica et al., 2006; Lupiáñez et al., 1997). Currently, in 3-D space, the facilitatory phase has been well established, yet it remains unclear how visuospatial attention orients/reorients in depth during the later inhibitory phase (IOR). To the best of our knowledge, only two relevant studies have investigated the spatial IOR effect in 3-D space. Theeuwes and Pratt (2003) used a variant to the classic Posner (1980) cueing task presented in 3-D environment and found that responses were equally slowed to targets at two different depth locations, so long as the targets shared the same cued location in the horizontal and vertical dimensions. Theeuwes and Pratt suggested that if a location in the horizontal $(x-y)$ plane is inhibited, the depth $(z)$ plane in front of and behind the cued location is inhibited, as well. In other words, IOR appears to spread across depth, and thus is not depth-specific. A subsequent study (Bourke, Partridge \& Pollux, 2006) largely followed that prior work, in that stimuli were presented at different horizontal locations and two different apparent depths through the use of stereoscopic disparity ( \pm 15 min of arc), but Bourke et al. increased the horizontal separation of stimuli that had virtually overlapped in the original study. Bourke et al. suggested that a small spatial IOR effect could be elicited by increasing the horizontal separation of stimuli that had virtually overlapped in the near and far depth planes. However, in that study they could not preclude the interpretation that the smaller IOR sizes were due to the two stimuli that shared the same position but different depths also having different horizontal distances, thus causing attentional orienting/reorienting in the horizontal plane to confound the results.

With regard to attentional reorienting theory, Klein (2000) suggested that IOR effects are observed only when attention disengages from the cued location. In spatial-cueing/IOR tasks, if there is no central cue (second cue), attention would not tend to disengage from the cued location. Therefore, the second cue location is rather important in spatial-cueing/IOR tasks. However, in the two prior studies, the second cue location (the fixation location) made the trajectory over which 
attention oriented/reoriented in depth nonstraight (e.g., in the uncued conditions, the left location in the near depth plane was cued, then the central fixation was cued to attract attention away from the previously cued location, and the target was presented at the left location, but in the far depth plane). Previous evidence has suggested that attention reorients mostly along a straight trajectory in the 2-D world. In comparison to a straight trajectory, the distance is larger when attention is reoriented along a nonstraight trajectory to the "novelty" stimuli, thus resulting in a slowing down of the speed toward the "novelty" stimuli, and a reduction in the amount of IOR (Bennett \& Pratt, 2001). Furthermore, attentional reorienting to fixation results in a significant reduction in the inhibitory effect (Pratt, O'Donnell, \& Morgan, 2000). Therefore, the null inhibitory effect in depth might have been caused by the paradigm in which the second cue was always the central fixation.

Whereas prior studies have focused largely on whether an IOR effect occurs when attention is oriented/reoriented in depth (Bourke et al., 2006; Theeuwes \& Pratt, 2003), in the present study we not only investigated whether the IOR effect is depth-specific, but also explored whether IOR effects were the same or different when attention was oriented/reoriented along different trajectories in 3-D space. As we know, IOR is considered to prevent attention or eye movement toward a previously examined location in order to improve the efficiency of visual search (Klein, 2000; Tipper et al., 1994). Given the characteristics of IOR in visual searches, together with the fact that we live in a real, 3-D world, it is necessary to investigate the spatial distribution of IOR in different hemispaces and at different depths in 3-D space. Chen et al. (2012) constructed a virtual 3-D world in an adapted version of the Posner spatial-cueing paradigm, investigating the facilitatory mechanisms underlying attention orienting/reorienting at different trajectories in 3-D space. They found that when attention was oriented/reoriented to the hemispace the cue pointed to in the valid depth condition, reaction times (RTs) were longer-similarly to when attention was oriented/reoriented in the uncued depth plane and to the spatial location in the opposed hemispace. However, although the same spatial distance was involved, attentional reorienting to objects unexpectedly appearing closer to the observer in the unattended hemispace was faster than reorienting to unexpected objects that were farther away. Furthermore, when attention was oriented/reoriented in the uncued depth plane but at the position in the same hemispace as the cue, RTs were shorter. These results suggest that the facilitatory effects are different when attention is oriented/reoriented along different trajectories in 3-D space. Therefore, in the present study, it was important not only to characterize the direction-specific mechanisms through which visuospatial attention is directed toward stimuli that require different trajectories in the later, inhibition phase, but also to determine whether the IOR effects are the same or different when attention is oriented/reoriented in different trajectories in 3-D space.

In Experiment 1, the Posner exogenous cueing paradigm was adapted to a virtual 3-D world, presenting targets either near to or far from participants and manipulating the validity of the cues to construct different attentional trajectories. For example, one cue would require attention to orient/reorient in the horizontal plane ( $x$-axis), and another would require orienting/reorienting in the depth plane (z-axis), in order to avoid the problems experienced in the previous two studies; a third cue would require orienting/reorienting in both the horizontal and depth planes ( $x$ - and $z$-axes). If IOR is depthspecific, attention could effectively orient/reorient in the depth plane (z-axis), resulting in RTs to targets at cued locations being longer than the RTs to targets at uncued locations. In contrast, if IOR is depth-blind, attention could not effectively orient/reorient in the depth plane ( $z$-axis), resulting in no difference between the RTs to targets at cued and uncued depth locations. In addition, the ecological importance of the 3-D world would influence the attentional orienting/reorienting, resulting in an interaction between attentional trajectory and target depth. Although the same set of 3-D objects (placeholders and targets) were used for both the near and far depth planes in Experiment 1, the retinal sizes of the objects were different in the near and far depth planes, a situation that could confound our results. To rule out this possibility, we conducted Experiment 2, a 2-D control experiment in which a group of new participants performed the same attentional orienting/ reorienting task described in Experiment 1. However, the task was performed under 2-D conditions in which the retinal sizes and retinal positions of the objects were identical to those in Experiment 1, though the depth dimension was removed. Hence, if Experiment 2 were to exhibit the same pattern of results as Experiment 1, the differential retinal images of objects, rather than their reorienting in depth, could have caused the results. In contrast, if the patterns of results differed between the two experiments, the results could most likely be attributed to the depth reorientation per se.

\section{Method}

\section{Participants}

Twenty-five participants (16 women, nine men; age: $22 \pm$ 1.8 years) participated in Experiment 1, and 25 participants (18 women, seven men; age: $21 \pm 2.5$ years) participated in Experiment 2. All participants were right-handed and had normal color vision and visual acuity, and none had a history of neurological or psychiatric disorders. All were paid for their participations after the experiment. This study was approved by the Academic Committee of the Department of Psychology, Soochow University, China. 


\section{Apparatus, stimuli, and experimental setup}

In Experiment 1, all stimuli were presented on a $27-$ in. ASUS 3-D monitor driven by an Nvidia GeForce FX 5200 graphics card. A pair of Nvidia 3-D shutter glasses synchronized with the monitor provided two separate stereoscopic displays to each of the two eyes, with a resolution of 800 (horizontal) $\times$ 600 (vertical) pixels at a refresh rate of $60 \mathrm{~Hz}$. All 3-D objects were presented on a black background that was custom-made using computer scripts (Presentation software package; Neurobehavioral Systems, Albany, CA).

The default virtual display in each trial consisted of three white placeholders in the near depth (sagittal) plane, three white placeholders in the mid depth plane, and three white placeholders in the far depth plane; thus, nine spatial locations in the virtual 3-D space were marked (see Fig. 1). The 3-D objects in the far depth plane appeared behind the monitor screen, whereas the 3-D objects in the near depth plane popped out of the monitor screen. Objects in the mid plane appeared to be on the screen surface, equal distances from the near and far depth planes. The different target distances were simulated by adjusting the binocular disparity. The binocular disparity between the near and far depth planes was $\pm 52.40 \mathrm{~min}$ of arc, relative to the fusion plane at which the central location in mid plane was presented (zero disparity). Participants reported that they could clearly perceive both the near and far depth planes when fixating the central location in the mid plane. The horizontal spatial distances (along the $x$ axis) between the left and right placeholders in the near and far depth planes were matched in visual angle $\left(16.85^{\circ}\right)$; that is, the retinal distances between the spatial positions were matched for the near and far depth planes. The 3-D objects in both depth planes were of the same perceived size, resulting in slightly different retinal sizes of the near and far stimuli: $1.98^{\circ}$ for the near and $1.52^{\circ}$ for the far objects. To prevent the far objects from being occluded by nearer objects, the stimuli in the near and far depth planes were slightly shifted so that the vertical retinal distance between the near and far positions was $2.36^{\circ}$ of visual angle. For half of the participants involved in the present study, the positions in the near depth plane were lower, and for the other half of participants, the near positions were higher. The target was a blue sphere, and the cue and the central cue consisted of transient flashes of one of the peripheral placeholders and one of the central placeholders, respectively.

In Experiment 2, participants sat in front of a monitor screen with no goggles, and 2-D stimuli were presented on the monitor screen with an eye-to-monitor distance of $75 \mathrm{~cm}$. The retinal sizes of the 2-D stimuli matched those of the 3-D stimuli in Experiment 1.

\section{Experimental procedures and design}

Experiment 1 in essence was a detection task. At the beginning of each trial, one peripheral location in either the near or the far depth plane was cued for $300 \mathrm{~ms}$, followed by a $200-\mathrm{ms}$ interstimulus interval. Subsequently, one location in the mid plane or the central location in either the near or the far depth plane was cued for $300 \mathrm{~ms}$, to attract attention away from the previously cued location. After another period of 150 or

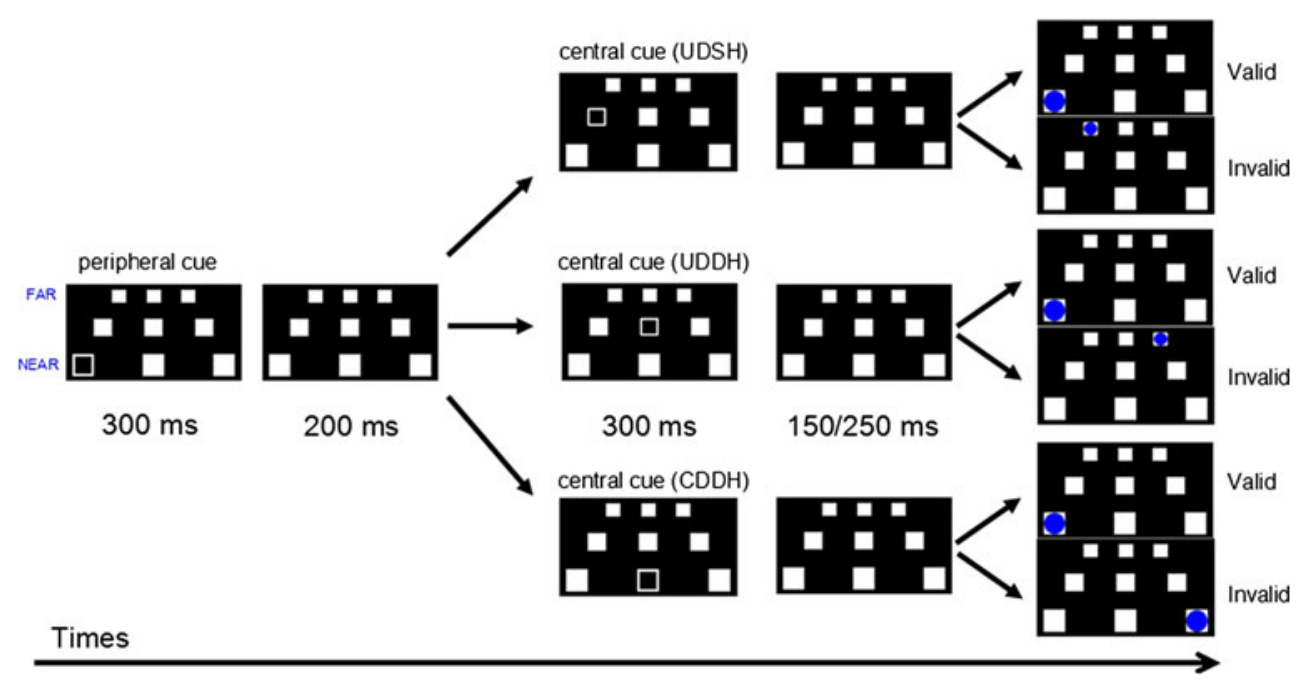

Fig. 1 Top view of exemplary trials of the experimental paradigm. The default visual scene consisted of nine white placeholders (three in the near depth plane, three in the midsagittal depth plane, and three in the far depth plane). The spatial distances among the three placeholders in the near, mid, and far depth planes had equal visual angles, and the same 3-D objects were presented in all three depth planes. All participants reported seeing the same-sized objects in the three depth planes because of the size-distance constancy effect (Boring, 1964). To prevent far objects from being occluded by near objects, the vertical distance between positions in the near and far depth planes was slightly shifted by $2.36^{\circ}$. The black placeholders represented the cues. The paths of overt attentional reorienting in 3-D space were cued depth, different hemispace (CDDH); uncued depth, same hemispace (UDSH); and uncued depth, different hemispace (UDDH) 
$250 \mathrm{~ms}$ (these two randomly arranged intervals were used to prevent temporal orienting), the target was presented for $250 \mathrm{~ms}$ at one of the peripheral locations in either the cued or the uncued depth plane, with equal probabilities. The three types of attentional trajectories were as follows: attention oriented/reoriented along the depth plane in which the cue was located, but in the opposite hemispace [i.e., cued depth, different hemispaces (CDDH)]; attention oriented/reoriented along the uncued depth plane, but in the same hemispace as the cue [i.e., uncued depth, same hemispace (UDSH)]; and attention oriented/reoriented not only along the uncued depth plane, but also in the opposite hemispace [i.e., uncued depth, different hemispaces (UDDH)]. The cue validity was crossed with the attentional trajectory, and the cues were valid in $50 \%$ the trials. Participants were instructed to fixate on the central fixation location in the midsagittal plane (zero disparity) throughout the experiment and to respond as quickly and accurately as possible to the target by pressing a key on the response box with their thumb. Prior to the formal test, each participant performed a practice session in which the composition of types of trials was the same as in the formal experiment.

The experimental, therefore, had a 2 (Target Depth: near vs. far) $\times 3$ (Attentional Trajectory: CDDH vs. UDSH vs. UDDH) $\times 2$ (Cue Validity: valid vs. invalid) within-subjects design, resulting in 12 experimental conditions in total. The experiment consisted of 624 trials, including 576 experimental trials (there were 48 trials in each condition) and 48 catch trials (to prevent participants from responding before the target onset, during which only the cues, and not the targets, were presented).

In Experiment 2, the experimental paradigm and task were the same as were described in Experiment 1, except that one independent variable was changed: The targets' retinal sizes replaced the target depth in Experiment 1. Therefore, this experimental had a 2 (target retinal size: small vs. large) $\times 3$ (attentional trajectory: CDDH vs. UDSH vs. UDDH) $\times 2$ (cue validity: valid vs. invalid) within-subjects design.

\section{Results}

\section{Experiment 1}

Incorrect responses (miss trials) and RTs beyond three standard deviations were discarded. Errors were below $2 \%$ across all of the conditions and were not analyzed further.

Table 1 provides a summary the mean RTs and standard errors under all conditions in Experiment 1. Correct RTs in the 12 experimental conditions were entered into a 2 (Target Depth: near vs. far) $\times 3$ (Attentional Trajectory: CDDH vs. UDSH vs. UDDH) $\times 2$ (Cue Validity: valid vs. invalid) repeated measures analysis of variance (ANOVA). The results showed that the main effect of attentional trajectory was significant, $F(2,48)=16.63, p<.001, \eta^{2}=.41$. Post-hoc comparisons (Bonferroni-corrected for multiple comparisons) showed that RTs were longer in the CDDH condition (365 ms) than in the UDDH condition (357 ms), $M D=7.81$, $p<.005$; longer in the UDSH condition $(372 \mathrm{~ms})$ than in the UDDH condition ( $357 \mathrm{~ms}$ ), $M D=14.46, p<.001$; and longer in the UDSH condition $(372 \mathrm{~ms})$ than in the CDDH condition (365 ms), $M D=6.65, p<.005$. The main effect of cue validity was also significant, $F(1,24)=72.65, p<.001, \eta^{2}=.75$, suggesting that the RTs to the targets at the cued location (375 ms) were longer than RTs to the targets at the uncued location (354 ms); however, the main effect of target depth was not significant, $F(1,24)=2.33, p>.05$. The interaction between target depth and cue validity was significant, $F(1,24)$ $=4.38, p<.05, \eta^{2}=.11$, as were the interaction between attentional trajectory and cue validity, $F(2,48)=8.46, p=$ $.001, \eta^{2}=.26$, and the three-way interaction between target depth, attentional trajectory, and cue validity, $F(2,48)=23.93$, $p<.001, \eta^{2}=.50$. However, the interaction between target depth and attentional trajectory was not significant, $F(2,48)=$ $2.96, p>.05$. To further test the potential differential interaction between cue validity and attentional trajectory in the near and far depth planes, a 2 (Cue Validity: cued vs. uncued) $\times 3$ (Attentional Trajectory: CDDH vs. UDSH vs. UDDH) repeated measures ANOVA was conducted for targets in the near and far depth planes (see Fig. 2).

For targets in the far depth plane, the main effect of attentional trajectory was significant, $F(2,48)=17.19, p<.001, \eta^{2}$ $=.42$. Post-hoc comparisons (Bonferroni-corrected for multiple comparisons) showed that RTs were longer in the CDDH condition $(365 \mathrm{~ms})$ than in the UDDH condition ( $357 \mathrm{~ms}$ ), $M D=8.52, p<.001$; longer in the UDSH condition ( $375 \mathrm{~ms}$ ) than in the UDDH condition ( $357 \mathrm{~ms}), M D=18.58$, $p<.001$; and longer in the UDSH condition (375 ms) than in the CDDH condition (365 ms), $M D=10.06, p=.005$. The main effect of cue validity was significant, $F(1,24)=54.75, p$ $<.001, \eta^{2}=.70$, suggesting that RTs to the targets at the cued location $(375 \mathrm{~ms})$ were longer than RTs to the targets at the uncued location $(357 \mathrm{~ms})$. Furthermore, the interaction between attentional trajectory and cue validity was also significant, $F(2,48)=22.00, p<.001, \eta^{2}=.48$. Additional test involving simple effects suggested that RTs were significantly longer to targets at cued locations than to those at uncued locations in the CDDH condition, $t(24)=9.17, p<.001$, and the UDDH condition, $t(24)=7.05, p<.001$. However, there was no significant difference between the RTs to targets at cued and uncued locations in the UDSH condition, $t(24)=$ $1.81, p>.05$. These results indicated that the typical IOR effects appeared only when attention was oriented/reoriented in the CDDH and UDDH conditions, whereas no IOR effect emerged when attention was oriented/reoriented in the UDSH condition. Comparing the IOR sizes in the $\mathrm{CDDH}$ and UDDH 
Table 1 Mean reaction times (RT) and standard errors ( $S E)$ of the means in the 3-D experiment and the 2-D control experiment (presented in the format $\mathrm{RT} \pm S E)$

\begin{tabular}{|c|c|c|c|c|c|c|}
\hline & \multicolumn{3}{|l|}{ Far } & \multicolumn{3}{|l|}{ Near } \\
\hline & Cued & Uncued & IOR & Cued & Uncued & IOR \\
\hline \multicolumn{7}{|l|}{ Experiment 1: 3-D Experiment $(N=25)$} \\
\hline Cued depth, different hemispace (CDDH) & $377 \pm 10$ & $354 \pm 10$ & 23 & $371 \pm 11$ & $357 \pm 11$ & 14 \\
\hline Uncued depth, same hemispace (UDSH) & $378 \pm 11$ & $373 \pm 11$ & 5 & $381 \pm 11$ & $355 \pm 10$ & 26 \\
\hline Uncued depth, different hemispace (UDDH) & $370 \pm 10$ & $344 \pm 10$ & 26 & $371 \pm 10$ & $343 \pm 10$ & 28 \\
\hline \multicolumn{7}{|l|}{ Experiment 2: 2-D Control Experiment $(N=25)$} \\
\hline Cued depth, different hemispace (CDDH) & $379 \pm 9$ & $351 \pm 7$ & 28 & $373 \pm 7$ & $350 \pm 7$ & 23 \\
\hline Uncued depth, same hemispace (UDSH) & $378 \pm 8$ & $352 \pm 7$ & 26 & $386 \pm 7$ & $365 \pm 7$ & 21 \\
\hline Uncued depth, different hemispace (UDDH) & $368 \pm 8$ & $340 \pm 7$ & 28 & $367 \pm 7$ & $345 \pm 7$ & 22 \\
\hline
\end{tabular}

Inhibition of return (IOR) is the difference between the valid and invalid conditions (in milliseconds)

conditions, we did not find any significant difference, $t(24)=$ $1.04, p>.05$.

For targets in the near depth plane, the main effect of attentional trajectory was significant, $F(2,48)=6.15, p<$ $.005, \eta^{2}=.20$. Post-hoc comparisons (Bonferroni-corrected for multiple comparisons) showed that RTs were longer in the CDDH condition $(365 \mathrm{~ms})$ than in the UDDH condition (357 ms), $M D=7.10, p<.05$, and longer in the UDSH condition $(368 \mathrm{~ms})$ than in the UDDH condition (357 ms), $M D=10.34, p=.005$. However, we found no difference between the RTs in the UDSH condition (368 ms) and the CDDH condition (364 ms), $M D=$ $3.24, p>.05$. The main effect of cue validity was significant, $F(1,24)=67.19, p<.001, \eta^{2}=.74$, suggesting that RTs to the targets at the cued location $(374 \mathrm{~ms})$ were longer than those to targets at the uncued location (352 ms). The interaction between attentional trajectory and cue validity was also significant, $F(2,48)=8.16, p$ $=.001, \eta^{2}=.25$. Additional tests involving simple effects suggested that RTs were significantly longer to targets at cued locations than to those at uncued locations in the CDDH condition, $t(24)=4.25, p<.001$; in the UDSH condition, $t(24)=7.38, p<.001$; and in the UDDH condition, $t(24)=7.41, p<.001$, thus indicating that typical IOR effects appeared regardless of the different

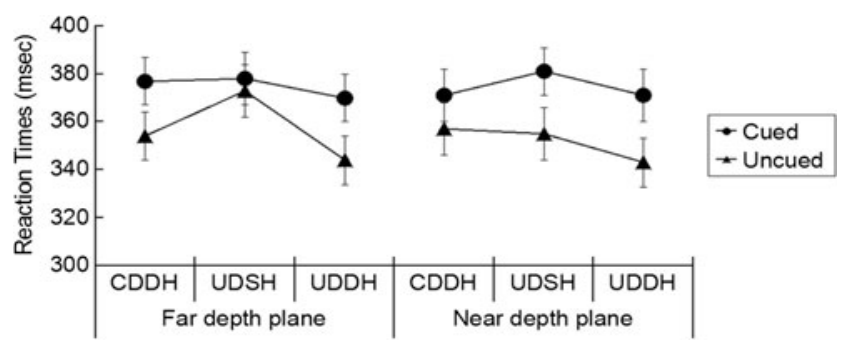

Fig. 2 Mean RTs, shown as a function of the experimental conditions in Experiment 1. The error bars represent SEMs trajectories in which attention was oriented/reoriented. According to the IOR effects from different attentional trajectories, however, we found that the IOR size was significantly smaller in the CDDH condition $(14 \mathrm{~ms})$ than in the UDSH condition (26 ms), $t(24)=3.20, p<.005$, or in the UDDH condition (28 ms), $t(24)=3.40, p<.005$. However, there was no significant difference between the IOR sizes in the UDSH and the UDDH condition, $t \mathrm{~s}<1$.

To summarize, these results indicate that attention oriented/reoriented in the UDSH condition differed between targets in the near and the far depth planes. Typical IOR effects were detected only when targets appeared in the near depth plane, whereas there was no IOR effect when targets appeared in the far depth plane. In addition, these results demonstrated that the IOR effects were the same when attention oriented/ reoriented along different trajectories when targets were detected in the near depth plane, even though the IOR size was smaller in the CDDH than in the UDSH and UDDH conditions.

\section{Experiment 2}

Incorrect responses and RTs beyond three standard deviations were discarded. Also, errors were below $2 \%$ across all conditions and were not analyzed further.

Table 1 presents a summary of the mean RTs and standard errors under all conditions in Experiment 2. Correct RTs were entered into a 2 (Target Retinal Size: small vs. large) $\times 3$ (Attentional Trajectory: CDDH vs. UDSH vs. UDDH) $\times 2$ (Cue Validity: valid vs. invalid) repeated measures ANOVA. The results showed that the main effect of attentional trajectory was significant, $F(2,48)=17.03, p<.001, \eta^{2}=.42$. Posthoc comparisons (Bonferroni-corrected for multiple comparisons) showed that RTs were longer in the $\mathrm{CDDH}$ 
condition $(363 \mathrm{~ms})$ than in the UDDH condition $(355 \mathrm{~ms})$, $M D=8.46, p<.001$; longer in the UDSH condition $(370 \mathrm{~ms})$ than in the UDDH condition $(355 \mathrm{~ms}), M D=$ $15.46, p<.001$; and longer in the UDSH condition (370 ms) than in the CDDH condition (363 ms), $M D=7.00, p<.05$. The main effect of cue validity was significant, $F(1,24)=$ $131.79, p<.001, \eta^{2}=.85$, thereby suggesting that RTs to targets at the cued location ( $375 \mathrm{~ms}$ ) were longer than RTs to targets at the uncued location $(351 \mathrm{~ms})$. However, the main effect of target retinal size was not significant, $F(1,24)=1.17$, $p>.05$. Moreover, all interactions were not significant, $F_{\mathrm{S}}<1$ (see Fig. 3).

According to these results, when we removed the depth dimension but retained the same object retinal sizes as in Experiment 1, all interactive effects disappeared, and typical IOR effects were presented regardless of the different attentional orienting/reorienting trajectories. These results clearly demonstrated that the differences when attention oriented/ reoriented along a UDSH trajectory in Experiment 1 were caused by attentional orienting/reorienting in depth between the near and far targets, rather than by the different retinal target sizes. ${ }^{1}$

\section{General discussion}

By incorporating the Posner exogenous cueing paradigm into a virtual 3-D world, we aimed to investigate whether spatial IOR effects are depth-specific and whether the spatial IOR effects are the same or different when attention is oriented/ reoriented along different trajectories in 3-D space. The results showed typical spatial IOR effects when attention was oriented/reoriented along the CDDH and UDDH trajectories, regardless of whether the targets appeared in the near or the far depth plane. However, the IOR effects differed between targets appearing in the near and far depth planes when attention was oriented/reoriented along the UDSH trajectory; typical spatial IOR effects were detected only when targets appeared in the near depth plane, and there was no spatial IOR effect when targets appeared in the far depth plane.

A prior study had shown that the spatial IOR effect is not depth-specific in 3-D space (Theeuwes \& Pratt, 2003), a finding that is in agreement with the viewpoint that attention does not operate in depth, or is "depth-blind" (Ghirardelli \& Folk, 1996; Iavecchia \& Folk, 1995). However, our results showed that spatial IOR was depth-specific when targets appeared in the near depth plane, whereas spatial IOR was not depthspecific when targets appeared in the far depth plane.

\footnotetext{
${ }^{1}$ We also conducted another 2-D control experiment, in which we turned off the emitter of the 3-D monitor but still asked the participants to wear the 3-D shutter glasses. We did this to verify whether the effects attributed to depth in Experiment 1 were in fact artifacts emerging from wearing the shutter goggles.
}

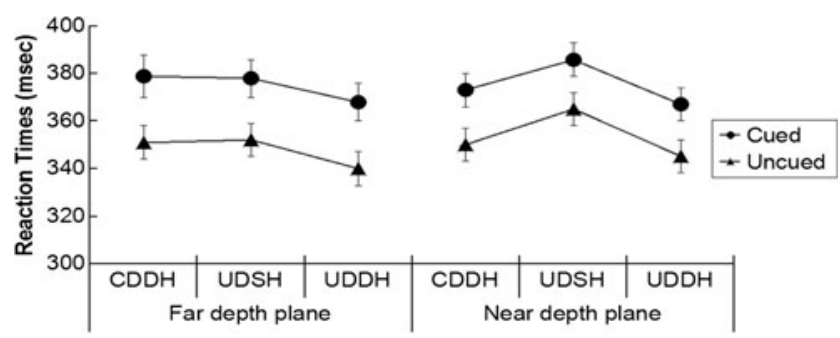

Fig. 3 Mean RTs, shown as a function of the experimental conditions in Experiment 2. The error bars represent SEMs

Furthermore, the results suggested that no effect was observed when attention was oriented/reoriented along the UDSH trajectory, an outcome that was not due to a faster response to the cued location, but rather to a slower response to the uncued location. ${ }^{2}$ These results also demonstrated that attentional reorienting to objects that unexpectedly appeared near observers in the UDSH condition was faster $(355 \mathrm{~ms})$ than reorienting to unexpected objects far away $(373 \mathrm{~ms}), t(24)=$ 5.23, $p<.001$. Therefore, we see that the former condition enlarged the IOR size, whereas the latter condition reduced the IOR size or removed the IOR effect completely. This finding was consistent with results indicating that responses to targets in the near plane are faster than responses to targets in the far plane (Theeuwes \& Pratt, 2003), and it is thus worth noting that asymmetrical effects have also been reported in previous studies (Andersen \& Kramer, 1993; Arnott \& Shedden, 2000; Atchley, Kramer, Andersen, \& Theeuwes, 1997). Recently, one study (Chen et al., 2012) showed that although the trajectories covered the same spatial distance, attentional reorienting to objects unexpectedly appearing near the observer and in the unattended hemispace occurred more quickly than did reorienting to unexpected far-away objects. Therefore, from an ecological perspective, while navigating the 3-D world, one of the most important behaviors involves the rapid redirection of our attention to potentially threatening or rewarding stimuli that unexpectedly invade the space near us, resulting in a reduction in RT. In contrast, reorienting to stimuli far from us - although it is also important in everyday life - is usually not so urgent, so that the RT is longer (de Gonzaga Gawryszewski et al., 1987; Graziano \& Cooke, 2006).

The critical difference between the present study and the prior study (Theeuwes \& Pratt, 2003) relies on the question of whether the spatial IOR effect is depth-specific. First, in the present study, we had attention orient/reorient along a straight trajectory in the depth plane (UDSH condition), in which the second cue location was no longer the central fixation location. A previous study had suggested that reorienting attention

\footnotetext{
${ }^{2}$ We also conducted a repeated experiment in which we included a neutral cue condition (using double cues), in order to reveal the extent to which that discrepant effect was due to inhibition at the cue versus response slowing at the uncued location.
} 
to a fixated location resulted in a significant reduction in the inhibitory effect (Pratt et al., 2000). Therefore, as compared with a straight trajectory, the distance when attention reoriented along a nonstraight trajectory to the "novelty" stimuli was larger, resulting in a slowing down of the speed toward the "novelty" stimuli and a reduction in the amount of IOR (Bennett \& Pratt, 2001). In addition, attentional momentum suggested that every trial included three movements of attention-from fixation to the cue, from the cue back to the fixation point, and from fixation to the target - and that orienting attention to the target is fastest when the third movement of attention has the same direction and amplitude as the second movement (Pratt, Adam, \& McAuliffe, 1998; Pratt, Spalek, \& Bradshaw, 1999). Therefore, the slowest detection responses occurred at the cued location, and the fastest responses occurred at the uncued location directly opposite the cued location (Bennett \& Pratt, 2001). In prior studies (Bourke et al., 2006; Theeuwes \& Pratt, 2003), the second cue was the central fixation, resulting in a trajectory of attentional orienting/ reorienting in the depth plane (i.e., the UDSH condition in the present study) that was not straight; instead, the third movement of attention exhibited different directions and amplitudes from the second movement when attention was oriented/ reoriented along the depth plane. Therefore, the slower responses at the uncued location either reduced the size of IOR or inhibited the IOR effect completely. Second, in the present study, the binocular disparity between the near and far depth planes was $\pm 52.40 \mathrm{~min}$ of arc, relative to the fusion plane at which the fixation cross was presented (i.e., zero disparity). These values are near the maximum disparity separation possible without loss of fusion, since a pilot experiment showed that trained observers had no trouble fusing the two images with a binocular disparity of $\pm 52.40 \mathrm{~min}$ of arc. In the prior studies (Bourke et al., 2006; Theeuwes \& Pratt, 2003), the binocular disparity between the near and far depth planes instead was \pm 15 min of arc relative to the fusion plane at which the fixation cross was presented. According to the gradient model of visual attention, the closer to the cued location, the stronger inhibition is; therefore, the perceived distances between the near and far depth planes were larger in the present study than in previous studies, resulting in the inhibition tending to spread across locations in same hemispace but different depths in the previous studies.

Although our results indicated that the IOR effects were different when attention was oriented/reoriented along different trajectories in 3-D space, the different spatial-cueing effects especially appeared between targets in the near and far depth planes when attention was oriented/reoriented in UDSH condition. One might argue that the pattern of our results might have arisen from a confound of the effects of depth and hemispace. That is, in the condition in which IOR was not found when targets were in the far depth plane, targets are presented at uncued depth, but also this was the only condition of the three in which the target appeared in the same hemispace (in the other two conditions-CDDH and UDSH - the target appeared in a different hemispace). In order to rule out this explanation, we suggest that, first, if that were true, it would not explain the significant effect in the same hemispace in the near target condition. Second, a previous study (Bennett \& Pratt, 2001) has shown that inhibition spreads beyond the cued location to affect the whole hemispace. However, this is likely only appropriate for 2-D space, in which the inhibition spreads along the $y$-axis. In the present study, the two locations at different depths in the same hemispace were at the same position on the $y$-axis, but at different positions on the $z$-axis. Therefore, that objection would not apply to the present study. Third, if inhibition could spread along the $z$-axis, according to the gradient model of visual attention, the closer to the cued location, the stronger inhibition is. The binocular disparity was $\pm 15 \mathrm{~min}$ of arc in previous studies (Bourke et al., 2006; Theeuwes \& Pratt, 2003), whereas the binocular disparity was $\pm 52.40 \mathrm{~min}$ of arc in the present study. Therefore, the perceived distances were larger in the present study than in previous studies, so inhibition only tended to spread to locations in the same hemispace but different depths in the previous studies.

Apart from the differences in how attention was oriented/ reoriented in the UDSH condition, consistent with the study by Theeuwes and Pratt (2003), we identified compatible IOR sizes between the UDDH condition $(26 \mathrm{~ms})$ and the CDDH condition $(23 \mathrm{~ms})$ when targets appeared in the far depth plane. According to our results, we speculated that although how attention was oriented/reoriented in the UDDH condition involved the depth dimension ( $z$-axis), the IOR size in this condition might mostly be attributed to attention orienting/ reorienting in the horizontal plane ( $x$-axis). Bourke et al. (2006) built upon prior work (Theeuwes \& Pratt, 2003) by increasing the horizontal separation of stimuli that had virtually overlapped, demonstrating that a small amount of spatial IOR operates within the depth plane (z-axis). In this case, the small amount of spatial IOR was obtained just when the near and far objects inhibited different positions in the horizontal plane ( $x$-axis). Therefore, when the targets appeared in the far depth plane in the present study, although the attentional trajectories varied between the UDDH and CDDH conditions, the IOR sizes were similar.

In addition, attention oriented/reoriented in the $\mathrm{CDDH}$ condition underwent some reduction in IOR size relative to the other two conditions (i.e., UDSH and UDDH) when targets appeared in the near depth plane. This reduction effect was perhaps surprising, yet it was not without an interpretation. When targets appeared at the uncued location in the near depth plane, both the UDSH and UDDH conditions involved one critical factor: When stimuli unexpectedly approach to us from a farther location, they instantaneously demand a shift in our attention toward them in order to secure survival (de 
Gonzaga Gawryszewski et al., 1987; Downing \& Pinker, 1985; Graziano \& Cooke, 2006; Previc, 1998). However, attentional orienting/reorienting in the $\mathrm{CDDH}$ condition did not demand a shift in our attention when stimuli were approaching from far away. Therefore, due to the different ecological importances of these attentional trajectories, the characterization of the directionspecific mechanisms of attentional orienting/reorienting varied.

Our results show that the depth-based spatial IOR is asymmetrical (e.g., in the UDSH condition), and we indicated that the ecological importance of the 3-D world would influence attentional orienting/reorienting. That is, behaviorally, fewer benefits exist for attentional reorienting from near to far locations than for attentional reorienting from far to near locations (Chen et al., 2012; de Gonzaga Gawryszewski et al., 1987; Downing \& Pinker, 1985; Maringelli et al., 2001), resulting in the former conditions reducing or removing the IOR effect, but the latter conditions enlarging the IOR effect. Neurally, some evidence has shown that the parietaloccipital junction (POJ) demonstrates a near-space preference in visuospatial processing in 3-D space, with the highest activation for near viewing and the lowest for far viewing (Cardin \& Smith, 2011; Quinlan \& Culham, 2007). Electrophysiological and tract tracting studies in monkeys have suggested a role of the visual area 6 (V6) complex as a potential candidate region in which information from the ventral and dorsal streams is integrated (Breveglieri, Galletti, Gamberini, Passarelli, \& Fattori, 2006; Breveglieri, Kutz, Fattori, Gamberini, \& Galletti, 2002; Galletti, Fattori, Kutz, \& Battaglini, 1997). In the human brain, the POJ is a putative homologue of the monkey V6 complex (Galletti, Gamberini, Kutz, Baldinotti, \& Fattori, 2005; Galletti, Kutz, Gamberini, Breveglieri, \& Fattori, 2003; Pitzalis et al., 2013). Therefore, stimuli that unexpectedly approach observers instantaneously demand a shift of attention toward them in order to secure survival. In contrast, stimuli far from observers may be less attention-demanding.

To summarize, by adapting the Posner spatial-cueing paradigm in a constituted virtual 3-D environment, we found that IOR effects were different along different trajectories in 3-D space and that there were asymmetrical IOR effects when attention was oriented/reoriented in the depth plane (i.e., the UDSH condition, in the present study). IOR was depth-specific when targets appeared in the near depth plane, but depth blindness occurred when targets appeared in the far depth plane. We suggest that IOR is not entirely "depth-blind," and that the ecological importance of the 3-D world influences the effects on IOR of different directional shifts of attention in 3-D space.
Author note Thanks are due W.F. and Y.L. for assistance with the present study. The work was supported by grants from Natural Science Foundation of China $(31371025,31371127)$. Q.C. is supported by the Program for New Century Excellent Talents in the University of China (NCET-12-0645) and by the Guangdong Province Universities and Colleges Pearl River Scholar Funded Scheme (2014). A.W. is supported by the Fundamental Research Funds for the Central Universities (14ZZ1202).

\section{References}

Andersen, G. J., \& Kramer, A. F. (1993). Limits of focused attention in three dimensional space. Perception \& Psychophysics, 53, 658-667. doi:10.3758/BF03211742

Arnott, S. R., \& Shedden, J. M. (2000). Attention switching in depth using random-dot autostereograms: Attention gradient asymmetries. Perception \& Psychophysics, 62, 1459-1473. doi:10.3758/ BF03212146

Atchley, P., Kramer, A. F., Andersen, G. J., \& Theeuwes, J. (1997). Spatial cuing in a stereoscopic display: Evidence for a "depthaware" attentional focus. Psychonomic Bulletin \& Review, 4, 524 529. doi:10.3758/BF03214343

Bennett, P. J., \& Pratt, J. (2001). The spatial distribution of inhibition of return. Psychological Science, 12, 76-80. doi:10.1111/1467-9280. 00313

Berlucchi, G., Chelazzi, L., \& Tassinari, G. (2000). Volitional covert orienting to a peripheral cue does not suppress cue-induced inhibition of return. Journal of Cognitive Neuroscience, 12, 648-663. doi: $10.1162 / 089892900562408$

Boring, E. G. (1964) Size constancy in a picture. The American Journal of Psychology, 77, 494-498.

Bourke, P. A., Partridge, H., \& Pollux, P. M. J. (2006). Additive effects of inhibiting attention to objects and locations in three-dimensional displays. Visual Cognition, 13, 643-654. doi:10.1080/ 13506280544000309

Breveglieri, R., Galletti, C., Gamberini, M., Passarelli, L., \& Fattori, P. (2006). Somatosensory cells in area PEc of macaque posterior parietal cortex. Journal of Neuroscience, 26, 3679-3684.

Breveglieri, R., Kutz, D. F., Fattori, P., Gamberini, M., \& Galletti, C. (2002). Somatosensory cells in the parieto-occipital area V6A of the macaque. NeuroReport, 13, 2113-2116.

Cardin, V., \& Smith, A. T. (2011). Sensitivity of human visual cortical area V6 to stereoscopic depth gradients associated with self-motion. Journal of Neurophysiology, 106, 1240-1249.

Cheal, M., \& Chastain, G. (2002). Timing of facilitatory and inhibitory effects of visual attention. Visual Cognition, 9, 969-1002.

Chen, Q., Weidner, R., Vossel, S., Weiss, P. H., \& Fink, G. R. (2012). Neural mechanisms of attentional reorienting in three-dimensional space. Journal of Neuroscience, 32, 13352-13362. doi:10.1523/ JNEUROSCI.1772-12.2012

Chica, A. B., \& Lupiáñez, J. (2009). Effects of endogenous and exogenous attention on visual processing: An inhibition of return study. Brain Research, 1278, 75-85.

Chica, A. B., Lupiáñez, J., \& Bartolomeo, P. (2006). Dissociating inhibition of return from the endogenous orienting of spatial attention: Evidence from detection and discrimination tasks. Cognitive Neuropsychology, 23,1015-1034. doi:10.1080/ 02643290600588277

Corbetta, M., Kincade, J. M., Lewis, C., Snyder, A. Z., \& Sapir, A. (2005). Neural basis and recovery of spatial attention deficits in spatial neglect. Nature Neuroscience, 8, 1603-1610.

Corbetta, M., Kincade, J. M., Ollinger, J. M., McAvoy, M. P., \& Shulman, G. L. (2000). Voluntary orienting is dissociated from target detection 
in human posterior parietal cortex. Nature Neuroscience, 3, 292297.

Corbetta, M., Patel, G., \& Shulman, G. L. (2008). The reorienting system of the human brain: From environment to theory of mind. Neuron, $58,306-324$.

Corbetta, M., \& Shulman, G. L. (2002). Control of goal-directed and stimulus-driven attention in the brain. Nature Review Neuroscience, 3, 201-215.

de Gonzaga Gawryszewski, L., Riggio, L., Rizzolatti, G., \& Umiltà, C. (1987). Movements of attention in the three spatial dimensions and the meaning of "neutral" cues. Neuropsychologia, 25, 19-29. doi: 10.1016/0028-3932(87)90040-6

Downing, C. J., \& Pinker, S. (1985). The spatial structure of visual attention. Cambridge, MA: MIT Press.

Galletti, C., Fattori, P., Kutz, D. F., \& Battaglini, P. (1997). Arm movement-related neurons in the visual area V6A of the macaque superior parietal lobule. European Journal of Neuroscience, 9, 410413.

Galletti, C., Gamberini, M., Kutz, D. F., Baldinotti, I., \& Fattori, P. (2005). The relationship between V6 and PO in macaque extrastriate cortex. European Journal of Neuroscience, 21, 959-970.

Galletti, C., Kutz, D. F., Gamberini, M., Breveglieri, R., \& Fattori, P. (2003). Role of the medial parieto-occipital cortex in the control of reaching and grasping movements. Experimental Brain Research, 153, 158-170.

Ghirardelli, T. G., \& Folk, C. L. (1996). Spatial cuing in a stereoscopic display: Evidence for a "depth-blind" attentional spotlight. Psychonomic Bulletin \& Review, 3, 81-86. doi:10.3758/ BF03210744

Graziano, M. S. A., \& Cooke, D. F. (2006). Parieto-frontal interactions, personal space, and defensive behavior. Neuropsychologia, 44, 845859. doi:10.1016/j.neuropsychologia.2005.09.009

Hayman, R., Verriotis, M. A., Jovalekic, A., Fenton, A. A., \& Jeffery, K. J. (2011). Anisotropic encoding of three-dimensional space by place cells and grid cells. Nature Neuroscience, 14, 1182-1190.

Iavecchia, H. P., \& Folk, C. L. (1995). Shifting visual attention stereo graphic displays: A time course analysis. Human Factors, 36, 606 618.

Janssen, P., Vogels, R., \& Orban, G. A. (2000). Selectivity for 3D shape that reveals distinct areas within macaque inferior temporal cortex. Science, 288, 2054-2056.

Kalogeropopulou, F., Woodruff, P. W. R., \& Vivas, A. B. (2015). Inhibition of return is not impaired but masked by increased facilitation in schizophrenia patients. Neuropsychology, 29, 10-16.

Klein, R. M. (2000). Inhibition of return. Trends in Cognitive Sciences, 4, 138-147. doi:10.1016/S1364-6613(00)01452-2

Lupiáñez, J., Decaix, C., Siéroff, E., Chokron, S., Milliken, B., \& Bartolomeo, P. (2004). Independent effects of endogenous and exogenous spatial cueing: Inhibition of return at endogenously attended target locations. Experimental Brain Research, 159, 447457.

Lupiáñez, J., Milán, E. G., Tornay, F. J., Madrid, E., \& Tudela, P. (1997). Does IOR occur in discrimination tasks? Yes, it does, but later. Perception \& Psychophysics, 59, 1241-1254. doi:10.3758/ BF03214211

Maringelli, F., McCarthy, J., Steed, A., Slater, M., \& Umiltà, C. (2001). Shifting visuo-spatial attention in a virtual three-dimensional space. Cognitive Brain Research, 10, 317-322.

Maruff, P., Yucel, M., Danckert, J., Stuart, G., \& Currie, J. (1999). Facilitation and inhibition arising from the exogenous orienting of covert attention depends on the temporal properties of spatial cues and targets. Neuropsychologia, 37, 731-744. doi:10.1016/S00283932(98)00067-0

Mayer, A. R., Seidenberg, M., Dorflinger, J. M., \& Rao, S. M. (2004). An event-related fMRI study of exogenous orienting: Supporting evidence for the cortical basis of inhibition of return? Journal of Cognitive Neuroscience, 16, 1262-1271. doi:10.1162/ 0898929041920531

Pitzalis, S., Sereno, M. I., Committeri, G., Fattori, P., Galati, G., Tosoni, A., \& Galletti, C. (2013). The human homologue of macaque area V6A. NeuroImage, 82, 517-530. doi:10.1016/j.neuroimage.2013. 06.026

Posner, M. I. (1980). Orienting of attention. Quarterly Journal of Experimental Psychology, 32, 3-25. doi:10.1080/ 00335558008248231

Posner, M. I., \& Cohen, Y. (1984). Components of visual orienting. In H. Bouma \& D. G. Bowhuis (Eds.), Attention and performance X: Control of language processes (pp. 531-556). Hillsdale, NJ: Erlbaum.

Pratt, J., \& Abrams, R. A. (1999). Inhibition of return in discrimination tasks. Journal of Experimental Psychology: Human Perception and Performance, 25, 229-242. doi:10.1037/0096-1523.25.1.229

Pratt, J., Adam, J., \& McAuliffe, J. (1998). The spatial relationship between cues and targets mediates inhibition of return. Canadian Journal of Experimental Psychology, 52, 213-216. doi:10.1037/ h0087294

Pratt, J., O'Donnell, C., \& Morgan, A. (2000). The role of the fixation location in inhibition of return. Canadian Journal of Experimental Psychology, 54, 186-195.

Pratt, J., Spalek, T. M., \& Bradshaw, F. (1999). The time to detect targets at inhibited and noninhibited locations: Preliminary evidence for attentional momentum. Journal of Experimental Psychology: Human Perception and Performance, 25, 730-746. doi:10.1037/ 0096-1523.25.3.730

Previc, F. H. (1998). The neuropsychology of 3-D space. Psychological Bulletin, 124, 123-164. doi:10.1037/0033-2909.124.2.123

Quinlan, D., \& Culham, J. (2007). fMRI reveals a preference for near viewing in the human parieto-occipital cortex. Neurolmage, 36, 167-187.

Sereno, M. E., Trinath, T., Augath, M., \& Logothetis, N. K. (2002). Three-dimensional shape representation in monkey cortex. Neuron, 33, 635-652.

Spence, C., Lloyd, D., McGlone, F., Nicholls, M. E. R., \& Driver, J. (2000). Inhibition of return is supramodal: A demonstration between all possible pairing of vision, touch and audition. Experimental Brain Research, 134, 42-48. doi:10.1007/s002210000442

Theeuwes, J., \& Pratt, J. (2003). Inhibition of return spreads across 3-D space. Psychonomic Bulletin \& Review, 10, 616-620. doi:10.3758/ BF03196523

Tipper, S. P., Weaver, B., Jerreat, L. M., \& Burak, A. L. (1994). Objectbased environment-based inhibition of return of visual attention. Journal of Experimental Psychology: Human Perception and Performance, 20, 478-499. doi:10.1037/0096-1523.20.3.478

Wright, R. D., \& Richard, C. M. (1996). Inhibition of return at multiple locations in visual space. Canadian Journal of Experimental Psychology, 50, 324-327.

Zhang, Y., Zhou, X., \& Zhang, M. (2012). Temporary inhibitory tagging at previously attended locations: Evidence from event-related potentials. Psychophysiology, 49, 1191-1199. 